\title{
Extracting features of Gaussian self-similar stochastic processes via the Bandt-Pompe approach
}

\author{
O. A. Rosso* \\ Chaos \& Biology Group, Instituto de Cálculo, Facultad de Ciencias Exactas y Naturales, Universidad de Buenos Aires (UBA), \\ Pabellón II, Ciudad Universitaria, 1428 Ciudad de Buenos Aires, Argentina \\ and Centre for Bioinformatics, Biomarker Discovery and Information-Based Medicine, School of Electrical Engineering \\ and Computer Science, The University of Newcastle, University Drive, Callaghan NSW 2308, Australia \\ L. Zunino ${ }^{\dagger}$ \\ Centro de Investigaciones Ópticas, C.C. 124 Correo Central, 1900 La Plata, Argentina \\ and Departamento de Ciencias Básicas, Facultad de Ingeniería, Universidad Nacional de La Plata (UNLP), 1900 La Plata, Argentina \\ D. G. Pérez \\ Instituto de Física, Pontificia Universidad Católica de Valparaíso (PUCV), 23-40025 Valparaíso, Chile \\ A. Figliola ${ }^{\S}$ \\ Chaos \& Biology Group, Instituto de Desarrollo Humano, Universidad Nacional de General Sarmiento, \\ Campus Universitario, Modulo 5, Juan Maria Gutierrez 1150, Los Polvorines, Pcia. de Buenos Aires, Argentina \\ H. A. Larrondo" \\ Facultad de Ingeniería, Universidad Nacional de Mar del Plata, Juan B. Justo 4302, 7600 Mar del Plata, Argentina \\ M. Garavaglia ${ }^{\text {II }}$ \\ Centro de Investigaciones Ópticas, C.C. 124 Correo Central, 1900 La Plata, Argentina \\ and Departamento de Física, Facultad de Ciencias Exactas, Universidad Nacional de La Plata (UNLP) \\ M. T. Martín ${ }^{* *}$ and A. Plastino ${ }^{\dagger \dagger}$ \\ Instituto de Física, Facultad de Ciencias Exactas, Universidad Nacional de La Plata (UNLP), C.C. 727, 1900 La Plata, Argentina \\ and Argentina's National Research Council (CONICET) \\ (Received 9 August 2007; published 12 December 2007)
}

\begin{abstract}
By recourse to appropriate information theory quantifiers (normalized Shannon entropy and Martín-PlastinoRosso intensive statistical complexity measure), we revisit the characterization of Gaussian self-similar stochastic processes from a Bandt-Pompe viewpoint. We show that the ensuing approach exhibits considerable advantages with respect to other treatments. In particular, clear quantifiers gaps are found in the transition between the continuous processes and their associated noises.
\end{abstract}

DOI: 10.1103/PhysRevE.76.061114

\section{INTRODUCTION}

Information theory concepts, like different entropic forms and statistical complexities [1-10], have proved to be useful quantifiers in the study and characterization of time series. In this work we will dwell on an often neglected point, namely, that in the evaluation of the above mentioned quantifiers, the determination of the underlying probability distribution $P$ (associated to a given dynamical system or time series) deserves detailed consideration. It is not a given. Indeed, prob-

\footnotetext{
*oarosso@ fibertel.com.ar

†lucianoz@ciop.unlp.edu.ar

\$dario.perez@ucv.cl

§afigliol@ungs.edu.ar

"larrondo@fi.mdp.edu.ar

I garavagliam@ ciop.unlp.edu.ar

mtmartin@fisica.unlp.edu.ar

†plastino@ fisica.unlp.edu.ar
}

PACS number(s): 02.50.-r, 02.50.Ey, 05.45.Tp, 05.40.-a ability distribution $P$ and sample space $\Omega$ are inextricably linked. Many schemes have been proposed for a proper selection of the probability space $(\Omega, P)$. We can mention, among others: (a) procedures based on amplitude statistics [11], (b) binary symbolic dynamics [12], (c) Fourier analysis [13], and (d) wavelet transform [14]. Their applicability depends on particular characteristics of the data such as stationarity, length of the time series, variation of the parameters, level of noise contamination, etc. In all these cases the global aspects of the dynamics can be somehow captured, but the different approaches are not equivalent in their ability to discern all the relevant physical details. One must also acknowledge the fact that the above techniques are introduced in a rather ad hoc fashion and are not directly derived from the system under study's dynamical properties themselves, as achieved, for instance, by the Bandt-Pompe methodology [15].

The Bandt-Pompe method (BPM) [15] for evaluating the probability distribution $P$ is based on the details of the attractor reconstruction procedure. Causal information is, consequently, properly incorporated into the construction pro- 
cess that yields $(\Omega, P)$. The Bandt-Pompe probability distribution is the only one among those in popular use that takes into account the temporal structure of the time series generated by the physical process under study. A notable result from the Bandt-Pompe approach is a notorious improvement in the performance of the information quantifiers obtained using the probability distribution $P$ generated by their algorithm $[10,16-18]$. Of course, one must assume with the BPM that the system is weakly stationary and that enough data are available for a correct attractor reconstruction.

In this work we revisit the characterization of two wellknown and widely used Gaussian self-similar stochastic processes: the fractional Brownian motion ( $\mathrm{fBm})$ and its noise, the fractional Gaussian noise (fGn). The former is a ubiquitous nonstationary model for many physical phenomena which have empirical spectra of power-law type, $1 / f^{\alpha}$, with $1<\alpha<3$. Thus, the characterization of these processes has become of interest in different and heterogeneous scientific fields, such as physics, biology, finance, and telecommunications [19-21]. We wish, in particular, to investigate the possibility of developing a unified framework for the description of the two stochastic processes. For this purpose we use the Bandt-Pompe approach so as to evaluate the all-important probability distribution that is associated to the time series generated by the two processes under analysis. We show that the ensuing procedure allows for the detection of important features of these Gaussian self-similar stochastic processes. A comparison with similar results, but obtained using probability distributions based on wavelet analysis, will provide us with an instructive counterpart [22] so as to properly assess what is to be gained with our present way of tackling things.

\section{INFORMATIONAL TOOLS}

\section{A. Normalized Shannon entropy and Martín-Plastino-Rosso intensive statistical complexity}

Several statistical complexity measures (SCM) have been recently introduced in the literature. These are the product of an entropic measure $\mathcal{H}$ times a distance (in probability space) to a fixed reference state, $\mathcal{Q}$ [5]. The latter quantity is usually called disequilibrium; it works as a quantifier of the degree of physical structure of a given time series. In Ref. [6] the disequilibrium $\mathcal{Q}$ was built up using Wootters' statistical distance and $\mathcal{H}$ was taken as the normalized Shannon entropy. The ensuing SCM is neither an intensive nor extensive quantity in the thermodynamical sense, although it yields useful results. A natural SCM improvement is to give it an intensive character, as in Ref. [7]. The concomitant SCM version is able to grasp essential details of the dynamics and capable of discerning among different degrees of periodicity and chaos. This measure, to be referred to as the MartínPlastino-Rosso (MPR) intensive statistical complexity, can be viewed as a functional $\mathcal{C}_{J S}[P]$ that characterizes the probability distribution $P$ associated with the time series generated by the dynamical system under study.
The MPR intensive statistical complexity measure is written as

$$
\mathcal{C}_{J S}[P]=\mathcal{Q}_{J}\left[P, P_{e}\right] \mathcal{H}_{S}[P] .
$$

It associates, to the probability distribution $P=\left\{p_{i}: i\right.$ $=1, \ldots, N\}$, the entropic measure (normalized Shannon entropy)

$$
\mathcal{H}_{S}[P]=S[P] / S_{\max }=\left(-\sum_{i=1}^{N} p_{i} \ln \left(p_{i}\right)\right) / S_{\max },
$$

where $\quad S_{\max }=S\left[P_{e}\right]=\ln N, \quad\left(0 \leqslant \mathcal{H}_{S} \leqslant 1\right) \quad$ and $\quad P_{e}$ $=\{1 / N, \ldots, 1 / N\}$ is the uniform distribution $-S$ stands for Shannon entropy. The disequilibrium $\mathcal{Q}_{J}$ is defined in terms of the extensive Jensen-Shannon divergence [7]. It reads

$$
\mathcal{Q}_{J}\left[P, P_{e}\right]=\mathcal{Q}_{0} \mathcal{J}\left[P, P_{e}\right],
$$

with

$$
\mathcal{J}\left[P, P_{e}\right]=\left\{S\left[\left(P+P_{e}\right) / 2\right]-S[P] / 2-S\left[P_{e}\right] / 2\right\},
$$

the above-mentioned Jensen-Shannon divergence, and

$$
\mathcal{Q}_{0}=-2\left\{\left(\frac{N+1}{N}\right) \ln (N+1)-2 \ln (2 N)+\ln N\right\}^{-1}
$$

a normalization constant, equal to the inverse of the maximum possible value of $\mathcal{J}\left[P, P_{e}\right]$, i.e., the value obtained when one of the components of $P$, say $p_{m}$, equals unity and the remaining $p_{i}$ vanish. This intensive quantity reflects on the systems's architecture, being different from zero only if there exist privileged, or more likely states among the accessible ones [5-7]. It quantifies not only randomness, but the presence of correlational structures as well $[6,7]$. The opposite extremes of perfect order and maximal randomness possess no structure to speak of. In between these two special instances, a wide range of possible degrees of physical structure exist, degrees that should be reflected in the features of the underlying probability distribution.

We insist on the fact that the above SCM is not a trivial function of the entropy, in the sense that, for a given $\mathcal{H}_{S}$ value, there exists a range of possible $\mathrm{SCM}$ values between a minimum $\mathcal{C}_{\text {min }}$ and a maximum $\mathcal{C}_{\text {max }}[5,8,23]$. Thus, evaluating the SCM provides one important additional information regarding the peculiarities of a probability distribution, not already carried by the entropy. This fact gets emphasized if one uses a Bandt-Pompe probability distribution. In order to study the time evolution of the statistical complexity measure, a diagram of $\mathcal{C}_{J S}$ versus $\mathcal{H}_{S}$ can be used, the MPR causality plane (in this case, $\mathcal{H}_{S}$ can be regarded as an arrow of time [24]). This kind of diagram has been used to study changes in a system's dynamics originated by modifications of some characteristic parameters $[5,7,10,18,22,23,25-28]$.

\section{B. Bandt-Pompe approach}

Additional improvements can be expected if one modifies the manner in which the underlying probability distribution is extracted, by a better consideration of the system's dynamics. For this purpose we follow the Bandt-Pompe approach 
[15]. Given a time series $\left\{x_{t}: t=1, \ldots, M\right\}$ and an embedding dimension $D>1$, one is interested in ordinal patterns of order $D[15,29,30]$ generated by

$$
s \mapsto\left(x_{s-(D-1)}, x_{s-(D-2)}, \ldots, x_{s-1}, x_{s}\right),
$$

that is, to each time $s$ we assign a $D$-dimensional vector that results from the evaluation of the time series at times $s, s$ $-1, \ldots, s-(D-1)$. Clearly, the greater the $D$ value, the more information about the past is incorporated into these vectors. By the ordinal pattern of order $D$ related to the time $s$ we mean the permutation $\pi=\left(r_{0}, r_{1}, \ldots, r_{D-1}\right)$ of $(0,1, \ldots, D$ $-1)$ defined by

$$
x_{s-r_{D-1}} \leqslant x_{s-r_{D-2}} \leqslant \cdots \leqslant x_{s-r_{1}} \leqslant x_{s-r_{0}} .
$$

In order to get a unique result we consider that $r_{i}<r_{i-1}$ if $x_{s-r_{i}}=x_{s-r_{i-1}}$. Thus, for all the $D$ ! possible permutations $\pi$ of order $D$, the probability distribution $P=\{p(\pi)\}$ is given by their relative frequency

$$
p(\pi)=\frac{N_{\text {card }}\{s \mid s \geqslant D, s \text { has ordinal pattern } \pi\}}{M-D+1},
$$

where $N_{\text {card }}$ is the cardinality of the set-roughly speaking, the number of elements in it.

Summing up, Bandt \& Pompe [15] proposed a method for evaluating the probability distribution $P$ associated to a given time series based precisely in the peculiar facets of the attractor reconstruction problem. In such a way, causal information became duly incorporated into the construction process that yields $(\Omega, P)$. The BPM considers a partition of the $D$-dimensional state space determined by the intersections of $D$ ! hyperplanes of $\mathrm{R}^{D}: x_{1}=x_{2}, \ldots, x_{1}=x_{D} ; x_{2}=x_{3}, \ldots, x_{2}$ $=x_{D} ; \ldots ; x_{D-1}=x_{D}$. Thus, each permutation $\pi$ of order $D$ can be associated with one of the connected pieces determined by the partition. In other words, an ordinal pattern represents one connected piece of $R^{D}$, and the union of all pieces is the total state space $\mathbb{R}^{D}$. The probability distribution $P$ of ordinal patterns is given by the frequency, in the attractor structure, of each piece (pattern); that is, $P$ is assigned by counting the times that the attractor visits each piece-see Eq. (8). In particular, if the attractor is symmetric with respect to the hyperplanes, all the connected pieces have the same frequency and thus the distribution of ordinal patterns is uniform: the attractor visits all the partition pieces with the same frequency. Consequently, the information provided by the time series so as to predict geometric locations of successive $D$ strings vanishes and the entropy is maximal $(N=D$ ! then $S_{\max }=\ln D !$ and $\left.\mathcal{H}_{S}=1\right)$. On the other hand, if the situation is such that the attractor remains always within just one of the connected pieces, one can predict with certainty, i.e., $\mathcal{H}_{S}=0$.

The advantages of the BPM reside in (a) its simplicity, (b) its robustness, and (c) its invariance with respect to nonlinear monotonous transformations. Also, this method provides an extremely fast computational algorithm. It can be applied to any type of time series (regular, chaotic, noisy, or experimental) [15]. Remark that for the applicability of this approach we need not to assume that the time series under analysis is representative of a low dimensional dynamical system. Of course, the embedding dimension $D$ plays an important role for the evaluation of the appropriate probability distribution, since $D$ determines the number of accessible states $D$ !, and tells us about the necessary length $M$ of the time series needed in order to work with a reliable statistics. In particular, Bandt \& Pompe suggest for practical purposes to work with $3 \leqslant D \leqslant 7$. Concerning this last point in all calculations reported here the condition $M \gg D$ ! is satisfied [18].

In this paper we evaluate the normalized entropy $\mathcal{H}_{S}$ and the MPR intensive statistical complexity measure $\mathcal{C}_{J S}$ using the permutation probability distribution $P=\{p(\pi)\}$, introduced in this section.

\section{FRACTIONAL BROWNIAN MOTION AND FRACTIONAL GAUSSIAN NOISE}

Fractional Brownian motion $(\mathrm{fBm})$ is the only family of processes which is Gaussian, self-similar, and endowed with stationary increments-see Ref. [22] and references therein. The normalized family of these Gaussian processes, $\left\{B^{H}(t), t>0\right\}$, is the one with $B^{H}(0)=0$ almost surely, i.e., with probability $1, \mathbb{E}\left[B^{H}(t)\right]=0$ (zero mean), and covariance given by

$$
\mathbb{E}\left[B^{H}\left(t_{1}\right) B^{H}\left(t_{2}\right)\right]=\frac{1}{2}\left(t_{1}^{2 H}+t_{2}^{2 H}-\left|t_{1}-t_{2}\right|^{2 H}\right)
$$

for $t_{1}, t_{2} \in \mathbb{R}$. Here $\mathbb{E}[\cdot]$ refers to the average computed with a Gaussian probability density. The power exponent $0<H$ $<1$ is commonly known as the Hurst parameter or Hurst exponent. These processes exhibit memory for any Hurst parameter except for $H=1 / 2$ as one realizes from Eq. (9). The $H=1 / 2$ case corresponds to classical Brownian motion and successive motion increments are as likely to have the same sign as the opposite, there is no correlation among them. Thus, Hurst's parameter defines two distinct regions in the interval $(0,1)$. When $H>1 / 2$, consecutive increments tend to have the same sign so that these processes are persistent. For $H<1 / 2$, on the other hand, consecutive increments are more likely to have opposite signs, thus these processes are antipersistent. Fractional Brownian motions are continuous but nondifferentiable processes (in the classical sense). As a nonstationary process, they do not possess a spectrum defined in the usual sense; however, it is possible to define a generalized power spectrum of the form

$$
\Phi_{B^{H}}(f) \propto \frac{1}{|f|^{\alpha}}
$$

with the exponent $\alpha=2 H+1,1<\alpha<3$.

The fractional Gaussian noise is introduced as the process $\left\{W^{H}(t), t>0\right\}$ obtained from the $\mathrm{fBm}$ increments (for discrete time)

$$
W^{H}(t)=B^{H}(t+1)-B^{H}(t) .
$$

This is a stationary Gaussian process with mean zero and covariance given by 


$$
\begin{aligned}
\rho(k)=\mathbb{E}\left[W^{H}(t) W^{H}(t+k)\right]= & \frac{1}{2}\left[(k+1)^{2 H}-2 k^{2 H}\right. \\
& \left.+|k-1|^{2 H}\right], \quad k>0 .
\end{aligned}
$$

Note that for $H=1 / 2$ all correlations at nonzero lags vanish and $\left\{W^{1 / 2}(t), t>0\right\}$ thus represents the white noise. The power spectrum associated to the fractional Gaussian noise is also given by Eq. (10), $\Phi_{W^{H}}(f) \propto 1 /|f|^{\alpha^{\prime}}$, but with $\alpha^{\prime}=2 H$ $-1,-1<\alpha^{\prime}<1$. In the following we will use this power-law behavior with different ranges for $\alpha$, for the two stochastic processes under analysis, gathering both into a unified framework. According to what values it adopts, the parameter $\alpha$ describes one or the other of the two mentioned processes.

\section{RESULTS AND DISCUSSION}

For simulating the $\mathrm{fBm}$ and fGn time series we adopt the Davies-Harte algorithm [31], as recently improved by Wood and Chan [32], which is both exact and fast. In this work ten time series, each one of length $M=2^{15}$ were analyzed, each series starting at a different initial condition. For the evaluation of the corresponding associated Bandt-Pompe probability distribution $[15,29,30]$, we chosen to work with an embedding dimension $D=6\left(6 ! \ll 2^{15}\right)$. Notice that the BPM is applicable to both the fBm and fGn processes [33]. The concomitant mean values plus the corresponding standard deviations of both the normalized Shannon entropy $\mathcal{H}_{S}$ and the MPR intensive statistical complexity measure $\mathcal{C}_{J S}$ are plotted in Fig. 1 as functions of the parameter $\alpha$.

Inspection of Fig. 1 allows one to gather that a clear different dynamical behavior is associated to the $\mathrm{fBm}$ and to the fGn. The fGn exhibits high entropic values, $0.97<\mathcal{H}_{S}<1.0$, together with low ones for the MPR complexity $0.0<\mathcal{C}_{J S}$ $<0.06$. This agrees with intuitive ideas concerning the notion of noise (remember that for a totally random process, or ideal noise, we have $\mathcal{H}_{S}=1$ together with $\mathcal{C}_{J S}=0$ ). Maximum of the normalized entropy and minimum of the complexity are attained at $\alpha=0$ (white noise). Notice also that for $|\alpha|$ $>0$ one observes decreasing (increasing) values for the entropy $\mathcal{H}_{S}\left(\mathcal{C}_{J S}\right)$. Thus both, persistent and antipersistent noises, are characterized by similar values of the two quantifiers. On the other hand, the $\mathrm{fBm}$ is always characterized by decreasing (increasing) values of $\mathcal{H}_{S}\left(\mathcal{C}_{J S}\right)$ as $\alpha$ grows. As the parameter $\alpha \rightarrow 3$ we observe larger standard deviations (SD) values. We attribute them to fluctuations associated to errors in the numerical simulations. This conclusion requires somewhat more elaboration. We repeated our calculations employing a time series of length $M=2^{12}$. We observe similar features as those of our previous calculations (depicted in Fig. 1). It should be stressed than larger SD values were found. Moreover, similar behaviors are also observed for both quantifiers, $\mathcal{H}_{S}$ and $\mathcal{C}_{J S}$, when smaller embedding dimensions, $D=4$ and $D=5$, are considered, as you can conclude from Fig. 2.

In Figs. 1 and 2 clear quantifiers gaps can be appreciated between fGn and $\mathrm{fBm}$ values, indicative of their distinct dynamical nature. Curiously enough, this is a new result. A recent approach that employs probability distributions based
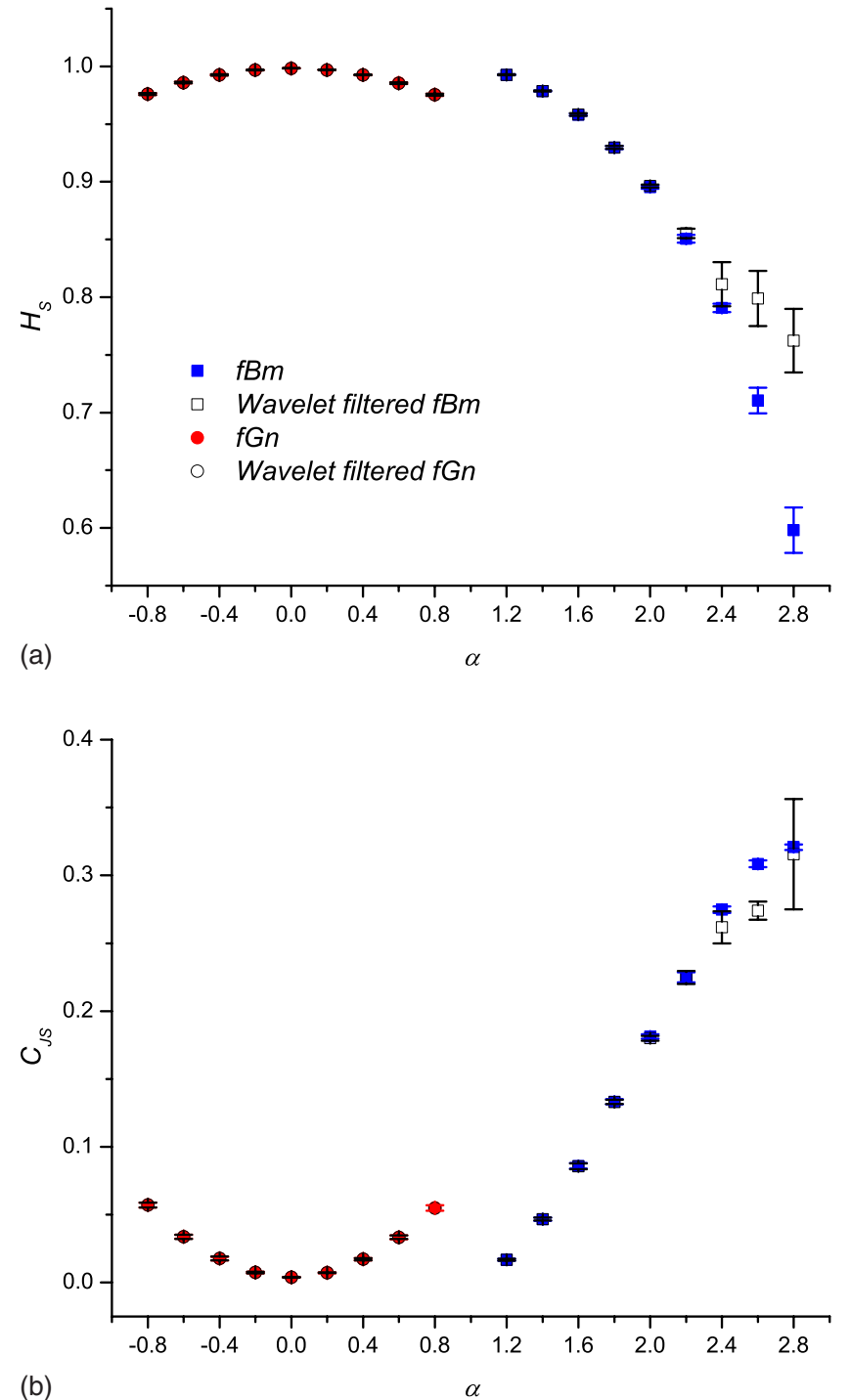

FIG. 1. (Color online) Mean values and SD corresponding to the normalized Shannon entropy $\mathcal{H}_{S}$ (top) and MPR intensive statistical complexity $\mathcal{C}_{J S}$ (bottom) as functions of the parameter $\alpha$ for both the original and the wavelet filtered time series.

on a wavelet description fails to notice such gaps [22]. For a fBm with $H \rightarrow 0(\alpha \rightarrow 1)$ we have also found a maximum of the normalized entropy $\left(\mathcal{H}_{S} \rightarrow 1\right)$ and minimum of the complexity $\left(\mathcal{C}_{J S} \rightarrow 0\right)$ in a similar way to the results obtained for a white noise. In order to ascertain whether we are here facing effects attributable to the way one obtains these probability distributions we repeated the above-described calculations for a wavelet filtered time series. That is, the orthogonal discrete wavelet transform (ODWT) that uses the spline cubic as a mother wavelet was obtained for each time series $[22,34,35]$, and then reconstructed by considering only the wavelet resolution levels $j=-9, \ldots,-1$. So, low frequency components are discarded. Obviously, our filtering process does modify long-range correlations. $\mathcal{H}_{S}$ and $\mathcal{C}_{J S}$ corresponding to these new, wavelet filtered, time series are also depicted in Fig. 1. The wavelet results are similar, for the fGn processes, to the previous ones. For the $\mathrm{fBm}$ processes they are different, however. This is due to the above-mentioned 

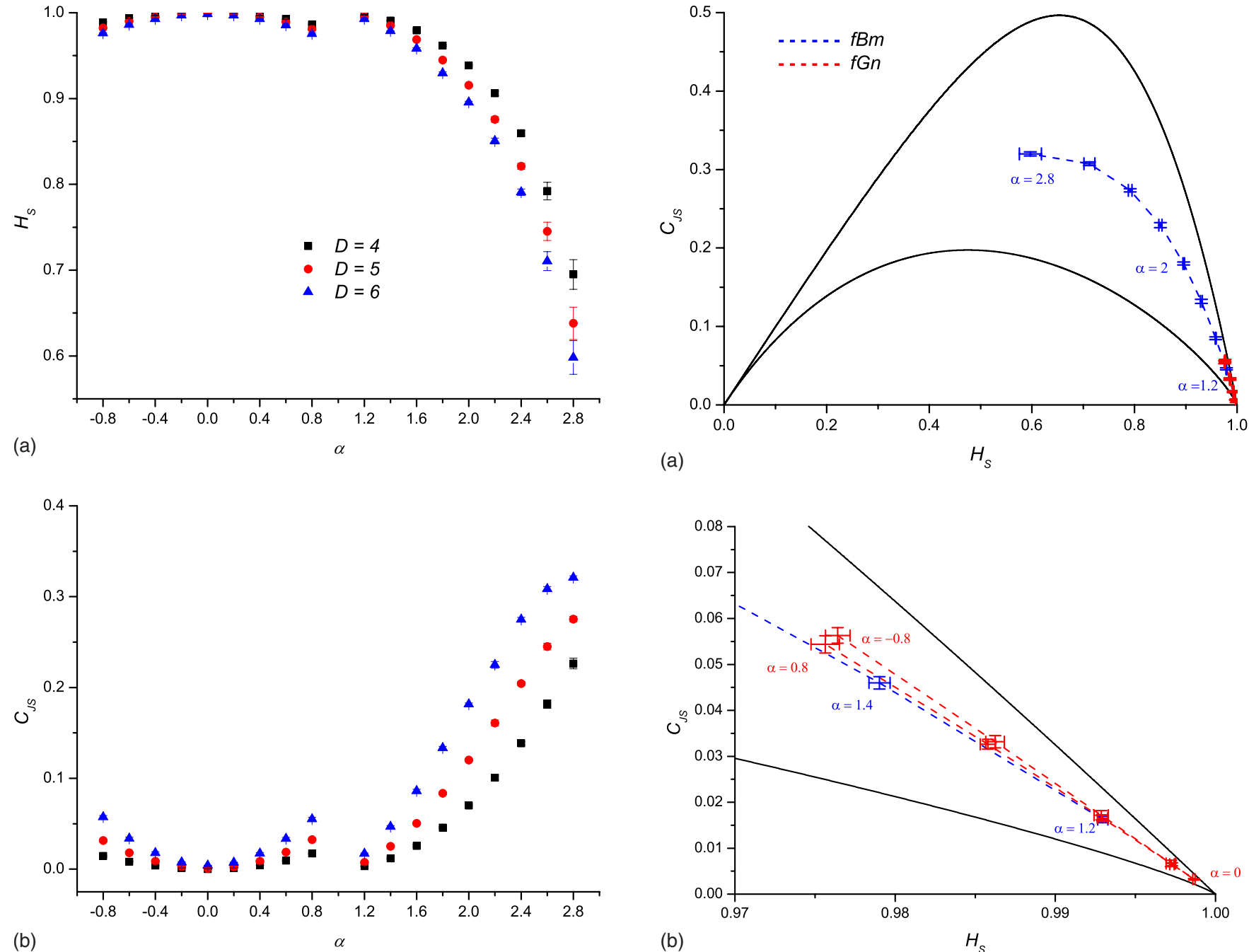

FIG. 2. (Color online) Mean values and SD corresponding to the normalized Shannon entropy $\mathcal{H}_{S}$ (top) and MPR intensive statistical complexity $\mathcal{C}_{J S}$ (bottom) as functions of the parameter $\alpha$ are plotted for our original time series with different embedding dimension $D$ $=4, D=5$, and $D=6$.

modifications in long-range correlations. Note that for $\alpha$ $\geqslant 2.2$ some differences are observed. We conclude that the clear different dynamical behavior associated to both processes ( $\mathrm{fBm}$ and $\mathrm{fGn}$ ), and more precisely the quantifiers gaps, are not an artifact of the BPM, they reflect on real features of these processes.

In Fig. 3 the two Gaussian self-similar stochastic processes are plotted in the MPR causality plane, described at the end of Sec. II A. Both processes are now located on the right-hand side of this plane. The fBm $(1<\alpha<3)$ exhibits entropic values in the interval $0.59<\mathcal{H}_{S}<1$ while the MPR complexity values are located in an almost equidistant fashion between the curves of maximum and minimum MPR complexity. Observe that the maximal and minimal values for the $\mathcal{H}_{S}$ are found for $\alpha=1.2$ and $\alpha=2.8$, respectively. As $\alpha$ increases, correlations among different values become apparent and, consequently, $\mathcal{H}_{S}$ decreases. Ordinary Brownian motion $(\alpha=2)$ is characterized by a relative high entropy and low MPR complexity $\left(\mathcal{H}_{S} \simeq 0.9\right.$ and $\left.\mathcal{C}_{J S} \simeq 0.18\right)$. Also, persis-

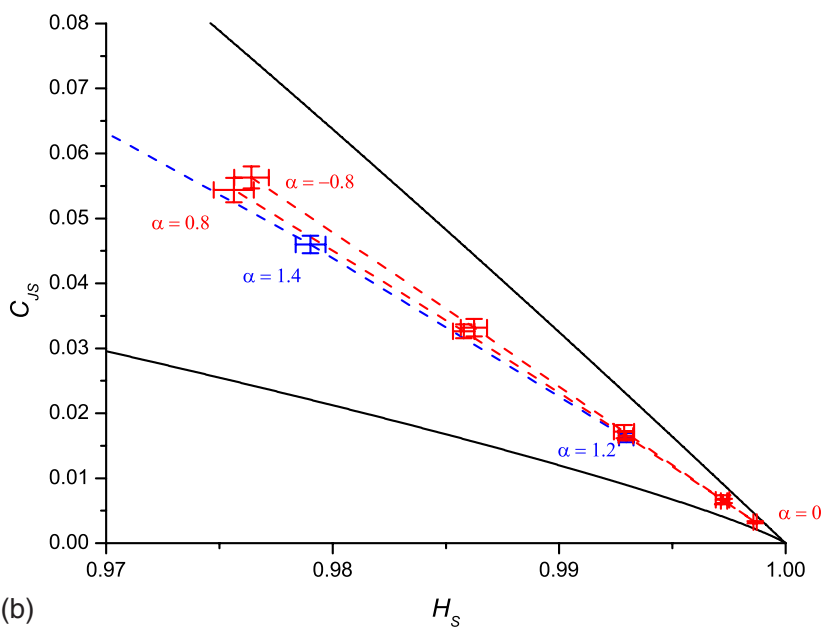

FIG. 3. (Color online) Continuous lines correspond to curves of minimum $\mathcal{C}_{\text {min }}$ and maximum $\mathcal{C}_{\max }$, respectively. Top: Localization of the fBm and fGn in the MPR causality plane. Bottom: Enlargement near the ideal point $\mathcal{H}_{S}=1, \mathcal{C}_{J S}=0$.

tent $\mathrm{fBm}(2<\alpha<3)$-long memory processes-are more complex than antipersistent $\mathrm{fBm}(1<\alpha<2)$-short memory ones-in agreement with the intuitive idea one has for this kind of behavior. Their associated noises $(-1<\alpha<1)$ exhibit higher entropic values $\left(0.97<\mathcal{H}_{S}<1\right)$ together with MPR complexity values between 0 and 0.06 . In particular, persistent and antipersistent $(|\alpha|>0)$ fGn display quite similar values. Maximum entropy and minimum MPR complexity values are observed for $\alpha=0$, which corresponds to white noise.

\section{CONCLUSIONS}

The focus of the present discussion has been the use of appropriate quantifiers for the characterization of relevant features of nonlinear dynamics in connection with the study of time series. In this work we dwelt on an often neglected point, namely, that for computing these quantifiers, the determination of the underlying probability distribution $P$ (associated to a given dynamical system or time series) is the 
basic starting stage that deserves detailed consideration. It is certainly not a given. There are several ways of obtaining it. We propose using the Bandt-Pompe methodology on account that the ensuing probability distribution arises in natural fashion, by taking into account the temporal structure of the time series generated by the physical process under study. Its advantages have here been reported with some detail and seem to recommend it. We wish here to emphasize just one notable Bandt-Pompe result with regards to the two quantifiers considered in this paper-see Figs. 1 and 2. Clear quantifiers gaps are detected in the transition between the two stochastic processes under analysis ( $\mathrm{fBm}$ and $\mathrm{fGn}$ ) that depend neither upon the length of the time series nor the embedding dimension. These gaps are not observed by using a wavelet approach [22].

\section{ACKNOWLEDGMENTS}

This work was partially supported by Consejo Nacional de Investigaciones Científicas y Técnicas (CONICET) (Grants PIP Nos. 0029/98, 5687/05, and 6036/05), Argentina, Comisión Nacional de Investigación Científica y Tecnológica (CONICYT) (FONDECYT Project No. 11060512), Chile, and Pontificia Universidad Católica de Valparaíso (PUCV) (Project No. 123.788/2007), Chile. O.A.R. gratefully acknowledges support from Australian Research Council (ARC) Centre of Excellence in Bioinformatics, Australia. The authors are very grateful to the reviewers, whose comments and suggestions helped to improve an earlier version of this paper.
[1] C. E. Shannon, Bell Syst. Tech. J. 27, 379 (1948); 27, 623 (1948).

[2] A. Rényi, Probability Theory (North-Holland Publishing Co., Amsterdam, 1970).

[3] C. Tsallis, J. Stat. Phys. 52, 479 (1988).

[4] J. S. Shiner, M. Davison, and P. T. Landsberg, Phys. Rev. E 59, 1459 (1999).

[5] R. López-Ruiz, H. L. Mancini, and X. Calbet, Phys. Lett. A 209, 321 (1995).

[6] M. T. Martín, A. Plastino, and O. A. Rosso, Phys. Lett. A 311, 126 (2003).

[7] P. W. Lamberti, M. T. Martín, A. Plastino, and O. A. Rosso, Physica A 334, 119 (2004).

[8] M. T. Martín, A. Plastino, and O. A. Rosso, Physica A 369, 439 (2006)

[9] D. G. Pérez, L. Zunino, M. T. Martín, M. Garavaglia, A. Plastino, and O. A. Rosso, Phys. Lett. A 364, 259 (2007).

[10] O. A. Rosso, H. A. Larrondo, M. T. Martín, A. Plastino, and M. A. Fuentes, Phys. Rev. Lett. 99, 154102 (2007).

[11] M. T. Martín, Ph.D. thesis, University of La Plata, La Plata, Argentina, 2004 (Spanish).

[12] K. Mischaikow, M. Mrozek, J. Reiss, and A. Szymczak, Phys. Rev. Lett. 82, 1144 (1999).

[13] G. E. Powell and I. C. Percival, J. Phys. A 12, 2053 (1979).

[14] O. A. Rosso and M. L. Mairal, Physica A 312, 469 (2002).

[15] C. Bandt and B. Pompe, Phys. Rev. Lett. 88, 174102 (2002).

[16] H. A. Larrondo, C. M. González, M. T. Martín, A. Plastino, and O. A. Rosso, Physica A 356, 133 (2005).

[17] H. A. Larrondo, M. T. Martín, C. M. González, A. Plastino, and O. A. Rosso, Phys. Lett. A 352, 421 (2006).
[18] A. M. Kowalski, M. T. Martín, A. Plastino, and O. A. Rosso, Physica D 233, 21 (2007).

[19] B. B. Mandelbrot, The Fractal Geometry of Nature (W. H. Freeman, New York, 1982).

[20] R. F. Voss, Phys. Rev. Lett. 68, 3805 (1992).

[21] P. Allegrini, M. Buiatti, P. Grigolini, and B. J. West, Phys. Rev. E 57, 4558 (1998).

[22] L. Zunino, D. G. Pérez, M. T. Martín, A. Plastino, M. Garavaglia, and O. A. Rosso, Phys. Rev. E 75, 021115 (2007).

[23] X. Calbet and R. López-Ruiz, Phys. Rev. E 63, 066116 (2001).

[24] A. R. Plastino and A. Plastino, Phys. Rev. E 54, 4423 (1996).

[25] A. M. Kowalski, M. T. Martín, A. Plastino, A. N. Proto, and O. A. Rosso, Phys. Lett. A 311, 180 (2003).

[26] A. M. Kowalski, M. T. Martín, A. Plastino, and O. A. Rosso, Int. J. Mod. Phys. B 19, 2273 (2005).

[27] O. A. Rosso, M. T. Martín, and A. Plastino, Physica A 347, 444 (2005).

[28] O. A. Rosso, M. T. Martín, A. Figliola, K. Keller, and A. Plastino, J. Neurosci. Methods 153, 163 (2006).

[29] K. Keller and H. Lauffer, Int. J. Bifurcation Chaos Appl. Sci. Eng. 13, 2657 (2003).

[30] K. Keller and M. Sinn, Physica A 356, 114 (2005).

[31] R. B. Davies and D. S. Harte, Biometrika 74, 95 (1987).

[32] A. T. A. Wood and G. Chan, J. Comput. Graph. Stat. 3, 409 (1994).

[33] C. Bandt and F. Shiha, J. Time Ser. Anal. 28, 646 (2007).

[34] D. G. Pérez, L. Zunino, M. Garavaglia, and O. A. Rosso, Physica A 365, 282 (2006).

[35] L. Zunino, D. G. Pérez, M. Garavaglia, and O. A. Rosso, Physica A 379, 503 (2007). 